\title{
Percutaneous foot joint needle placement using a C-arm flat-panel detector CT
}

\author{
Martin Wiewiorski • Martin Thanh Long Takes • \\ Victor Valderrabano • Augustinus Ludwig Jacob
}

Received: 15 February 2011 / Accepted: 30 May 2011 / Published online: 19 June 2011

(c) CARS 2011

\begin{abstract}
Purpose Image guidance is valuable for diagnostic injections in foot orthopaedics. Flat-detector computed tomography (FD-CT) was implemented using a $\mathrm{C}$-arm, and the system was tested for needle guidance in foot joint injections.

Methods FD-CT_-guided joint infiltration was performed in 6 patients referred from the orthopaedic department for diagnostic foot injections. All interventions were performed utilising a flat-panel fluoroscopy system utilising specialised image guidance and planning software. Successful infiltration was defined by localisation of contrast media depot in the targeted joint. The pre- and post-interventional numeric analogue scale (NAS) pain score was assessed.

Results All injections were technically successful. Contrast media deposit was documented in all targeted joints. Significant relief of symptoms was noted by all 6 participants.

Conclusions FD-CT-guided joint infiltration is a feasible method for diagnostic infiltration of midfoot and hindfoot joints. The FD-CT approach may become an alternative to commonly used 2D-fluoroscopically guidance.
\end{abstract}

Keywords Intra-articular injection - Image guided intervention · Flat-detector computed tomography

M. Wiewiorski $(\bowtie) \cdot$ V. Valderrabano

Orthopaedic Department, University Hospital of Basel,

Spitalstrasse 21, 4031 Basel, Switzerland

e-mail: mwiewiorski@uhbs.ch

M. T. L. Takes · A. L. Jacob

Institute of Interventional Radiology,

University Hospital Basel,

Basel, Switzerland

\section{Introduction}

It is a common procedure to infiltrate painful foot and ankle joints with local anaesthetics to determine the exact pain localization in degenerative joint conditions $[1,2]$. Such diagnostic intraarticular infiltrations are routinely performed under fluoroscopical guidance and proved to be useful in preoperative planning for predicting a successful outcome of operative procedures $[3,4]$. In the foot, infiltrations are often difficult due to the complex anatomy with small, tightly neighbouring joints [5]. In degenerative joint conditions, the accuracy is additionally often compromised by the presence of osteophytes and unusual post-traumatic or post-operatively disordered anatomy [6]. The radiation exposure that comes along with the fluoroscopical guidance should not be underestimated and relies on the experience of the performer [7]. Therefore, a feasible, precise, and safe infiltration technique for the foot and ankle is desirable, which can easily be implemented into a clinical setting.

Flat-detector computed tomography (FD-CT) has become widely accepted for interventional imaging using $\mathrm{C}$-arm systems, providing an efficient way of combining two-dimensional (2D) radiographic or fluoroscopic and 3D-CT imaging [8]. As for the musculoskeletal field of application, FD-CT has been successfully utilised for spinal interventions such as kyphoplasty or vertebroplasty $[9,10]$.

In this study, we present our first experience with FD-CT for infiltration of foot joints and evaluate its feasibility and potential use in routine preoperative diagnostic assessment.

\section{Material and methods}

Because the aim of this study was to assess the feasibility of a new technique, the number of admitted patients was 
restricted. The study population consisted of 6 consecutive patients ( 4 women, 2 men; mean age 46, range 36-58) admitted from the orthopaedic ambulance with unilateral chronic foot pain between May and July 2010.

Preinterventional clinical examination by an experienced orthopaedic surgeon (VV) included localization of the painful joint and pain status measured by a numeric analogue scale (NAS, $0=$ total absence of pain, $10=$ maximum imaginable pain [11]). All patients previously underwent diagnostic procedures consisting of planar radiographs and three phase ${ }^{99 \mathrm{~m}} \mathrm{Tc}-$ Dicarboxypropandiphosphate (DPD) single photon emission computed tomography-computed tomography (SPECT-CT) of the foot. SPECT-CT is part of a routine algorithm for diagnostics of degenerative joint disease of the foot and ankle. Two experienced radiologists (ALJ, MTLT) assessed the intensity and localisation of ${ }^{99 \mathrm{~m}} \mathrm{Tc}$-DPD-uptakes on SPECT-CT and defined the site of infiltration (Fig. 1a). Diagnoses included osteoarthritis (4 joints) and osteochondral lesions (2 joints) totalling 6 joints ( 2 talonavicular, 2 subtalar, 2 tarsometatarsal). Patients were referred to the interventional radiology department for diagnostic joint infiltration as part of a preoperative diagnostic assessment.

All interventions were performed with a flat-panel fluoroscopy system (ArtisZee, Siemens, Erlangen, Germany).

After positioning the patient on the fluoroscopy table, the target foot was being immobilized using a vacuum mattress (Fig. 1b). An initial scout view was performed according to preoperative imaging. Based on this overview, the target area was scanned to acquire a set of planning images. For this, the $\mathrm{C}$-arm with the flat-panel detector rotates $240^{\circ}$ around the target volume, creating a three dimensional dataset that allows interactive planning of the needles trajectory with the designated software (Syngo iGuide, Vers. 13, Siemens, Erlangen, Germany) by defining the insertion point on the skin and the target point in the joint (Fig. 1c).

At this point, sterile preparation of the skin including a sterile cover of the foot was conducted. The C-arm automatically drives along the ray path into position and the laser cross attached to the flat detector targets the skin entry site of the needle. The table position needs to be adjusted manually until the correct position is reached. This is controlled by an interactive bull's-eye view on the examination monitor, where a notch and a bead sight (one represents the table, the other the flat detector position) need to be superimposed on the other. The desired needle track can be easily estimated by holding the needles tip over the laser marked skin entry site and then moving the syringe until the laser cross is projecting on the end of the piston. Along the planned trajectory, local subcutaneous anaesthesia was applied (Mepivacain 1.5\%, Syntetica, Switzerland). A disposable needle (27 gauge, $25 \mathrm{~mm}$ long, Sterican, Braun Medical, Sempach, Switzerland) was used to enter the joint in all cases. The iGuide software offers an interactive support for steering the needle. By driving the $\mathrm{C}$-arm into oblique positions, the needles deviation to the planned trajectory are shown on the monitor and can be corrected until the target point is reached.

Following the placement of the needle, a control scan was performed to determine the position of the needle tip. If the correct placement of the needle was confirmed, $3 \mathrm{ml}$ of local anaesthetic (Bupivacain 0,5\%, Syntetica, Switzerland) mixed with iodine solution (Iopamiro 300, Bracco, Switzerland) in 2:1 ratio was injected into the joint.

After successful injection, a final image acquisition was carried out to document the exact localisation of the deposit (Fig. 1d).

The NAS outcome was assessed directly post-infiltration. Pain relief in responders was defined as reduction of NASscore of more than $50 \%$ of the preinterventional score.

The radiation dose was recorded in the RIS (INORIS, Nexus AG, Villingen-Schwenningen, Germany).

The study was approved by the institutional review board and written patient consent was obtained. The data were analysed by Student's t-test with a significance level of $P<0.05$ using the SPSS software (version 11.5.1, SPSS Inc., Chicago IL, USA).

\section{Results}

FD-CT - guided joint infiltration was successfully performed in all 6 joints without intra- or post-interventional complications. Iodine solution deposit was confirmed in all target joints. No intervention abort or conversion to planar fluoroscopy guided technique was necessary. In two patients, the foot had to be repositioned and the procedure re-planed after the primary referencing and trajectory planning procedure. This was due to patient movement on the fluoroscopy table that resulted in a discrepancy between the planned trajectory and actual position of the foot.

A pain relief was noted in all patients. The mean preinterventional NAS of 5.4 (range, 3-9; SD 2.2) decreased to a mean post-interventional NAS of 1.0 (range, $0-3$; SD 1.35). This was statistically significant $(P<0.01)$

The radiation dose for each patient is shown in Table 1. The radiation dose for patient 5 could not be estimated due to technical issues (data corruption).

\section{Discussion}

We present our first clinical experiences and results of the FD-CT - guided joint infiltration of foot joints. All patients showed an iodine solution deposit in the targeted joint and additionally significant pain relief after infiltration. 
(a)

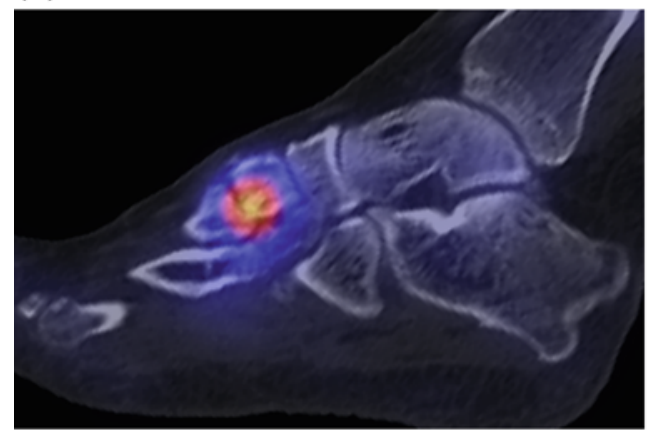

(c)

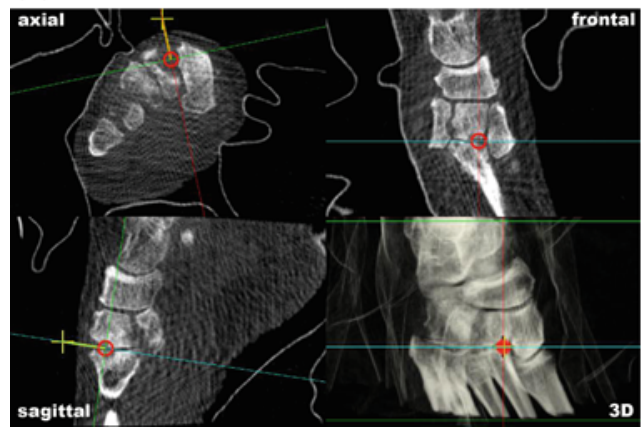

(b)

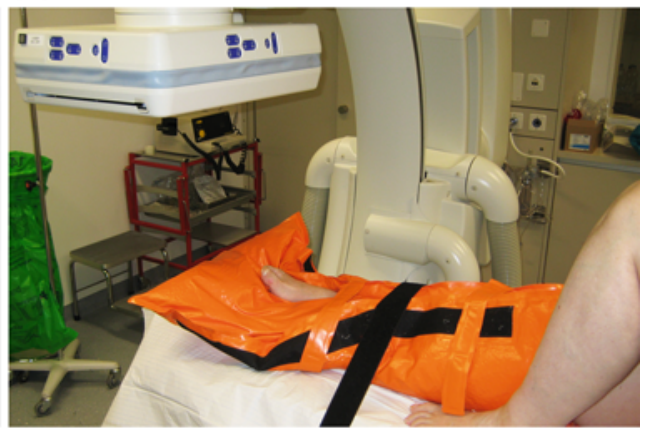

(d)

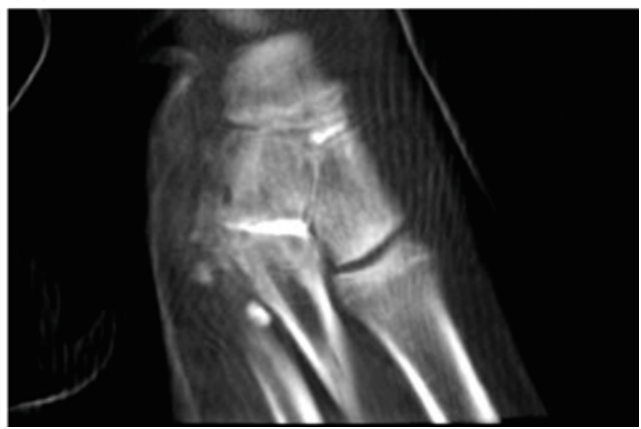

Fig. 1 Exemplar case. The patient presents with a painful midfoot (VAS 6) in the outpatient clinic. Preinterventional SPECT-CT shows joint degeneration with an increased pathological uptake in the second tarsometatarsal joint (TMT) (a). The foot is immobilized using a vacuum mattress (b). The needle trajectory is planned within multiplanar reconstructions by defining the insertion and target point within the acquired data set (c). After injection, a final image acquisition is carried out to document the exact localization of the deposit in the second TMT joint (d)

Table 1 Radiation dose

n.a. not available

\begin{tabular}{lllll}
\hline Patient & $\begin{array}{l}\text { Absorbed dose } \\
\left(\mathrm{cGy} \text { per } \mathrm{cm}^{2}\right)\end{array}$ & $\begin{array}{l}\text { Effective dose } \\
(\mathrm{mSv})\end{array}$ & $\begin{array}{l}\text { Length of procedure } \\
(\mathrm{min})\end{array}$ & $\begin{array}{l}\text { Reposition needed } \\
\text { (number) }\end{array}$ \\
\hline 1 & 111.9 & 0.02 & 35 & No \\
2 & 114.1 & 0.02 & 38 & No \\
3 & 113 & 0.02 & 33 & No \\
4 & n.a. & n.a. & 52 & Yes (1) \\
5 & 90.9 & 0.02 & 38 & No \\
6 & 310 & 0.06 & 54 & Yes (1) \\
Mean & 148.0 & 0.028 & 41.7 & \\
\hline
\end{tabular}

To the knowledge of the authors, this is the first description of this technique for foot joint infiltration.

Several authors describe good results of fluoroscopy guided needle placement in the midfoot and the subtalar joint [2-4], but such interventions can be difficult to perform in degenerative joint conditions $[5,12]$. To improve accuracy in such cases, CT-guidance proved to be useful. Saifuddin et al. reported technically successful foot and ankle intervention in 28 cases. Here, CT-guidance proved to be of special value for patients with post-traumatic, disordered anatomy and presence of osteophytes after failure of conventional fluoroscopically guided infiltrations [6]. Wiewiorski and al. described a CT-guided robot-assisted technique and successfully infiltrated 16 foot and ankle joints with degenerative joint disease [13]. Cases in which fluoroscopical guidance failed and successful conversion to CT-guided infiltration technique was performed have been also described by Mitchell et al. [2].

All 6 joints infiltrated in this study showed radiological signs of degeneration; however, severe deformation or extensive osteophytes were not found. Further studies will need to be performed to assess the usefulness of the described FD-CT guided technique for infiltration of severely distorted joints in comparison with fluoroscopy an CT. 
The mean effective dose for the intervention was calculated to be $0.028 \mathrm{mSv}$. Unfortunately, no radiation dose was mentioned in any of the publication regarding fluoroscopically or CT-guided foot infiltration [2,6,13]. Therefore, a comparison is not possible. As a point of reference, a SPECT of the foot has an effective dose of around $4.3 \mathrm{mSv}$.

To eliminate radiation exposure, sonography guided infiltrations of foot joints have been reported [14]. However, its usefulness in joints with an unphysiological joint anatomy, such as it is the case in osteoarthritis (presence of osteophytes), is limited. In a previous study, Berliner et al. showed that flat-panel detector systems can produce high quality radiographic images at a substantially reduced radiation dose in comparison with conventional computed radiography [15]. Future studies will have to evaluate the role of flat-detector guided imaging for routine interventions in terms of reduction of radiation exposure.

Certain limitations have been identified. A very important pre-condition for a successful FDCT_-guided infiltration is the strict immobilization of the patient's foot. Since this is a modality based intervention [16], even small changes in position of the foot lead to a discrepancy between initially acquired planning images and the actual position of the patient's foot, as seen in two of our patients. After reposition of the foot, a new planning scan has to be performed resulting in longer total intervention time (see patient 4 and 6 in Table 1) and increased radiation exposure (see patient 6 in Table 1). To address this issue, the authors plan to design an improved immobilizing device for future studies.

\section{Conclusion}

The first clinical cases demonstrated flat-detector computed tomography to be a feasible method for diagnostic infiltration of foot joints. Planning of needle placement in multiplanar reconstructions greatly shows good accuracy of intra-articular needle placement and allows exact and reliable application of local anaesthetics for preoperative diagnostic assessment. Although fluoroscopically guided injection remains the technique of choice, FD-CT is a reliable alternative.

Certain limitations were identified and require further experience in view of team experience, decrease in intervention time, radiation exposure and system modifications.

Future studies need to evaluate the usefulness of this novel technique for infiltration of joints in the presence of severe degenerative joint conditions (deformed joints and large osteophytes).

Conflict of interest There is no financial and personal relationships with other people or organisations that could inappropriately influence (bias) our work.
There is no employment, consultancies, stock ownership, honoraria, paid expert testimony, patent applications/registrations, and grants or other funding with other people or organisations involved in this manuscript.

\section{References}

1. Kretzschmar M, Wiewiorski M, Rasch H, Jacob AL, Bilecen D, Walter MA, Valderrabano V (2010) (99m)Tc-DPD-SPECT/CT predicts the outcome of imaging-guided diagnostic anaesthetic injections: a prospective cohort study. Eur J Radiol. doi:10.1016/j. ejrad.2010.09.013

2. Mitchell MJ, Bielecki D, Bergman AG, Kursunoglu-Brahme S, Sartoris DJ, Resnick D (1995) Localization of specific joint causing hindfoot pain: value of injecting local anesthetics into individual joints during arthrography. AJR Am J Roentgenol 164(6): 1473-1476

3. Khoury NJ, el-Khoury GY, Saltzman CL, Brandser EA (1996) Intraarticular foot and ankle injections to identify source of pain before arthrodesis. AJR Am J Roentgenol 167(3):669-673

4. Lucas PE, Hurwitz SR, Kaplan PA, Dussault RG, Maurer EJ (1997) Fluoroscopically guided injections into the foot and ankle: localization of the source of pain as a guide to treatment-prospective study. Radiology 204(2):411-415

5. Jones A, Regan M, Ledingham J, Pattrick M, Manhire A, Doherty M (1993) Importance of placement of intra-articular steroid injections. Bmj 307(6915):1329-1330

6. Saifuddin A, Abdus-Samee M, Mann C, Singh D, Angel JC (2005) CT guided diagnostic foot injections. Clin Radiol 60(2):191-195

7. Grainger AJ (2005) Image guidance of foot joint injections. Clin Radiol 60(2):189-190

8. Kalender WA, Kyriakou Y (2007) Flat-detector computed tomography (FD-CT). Eur Radiol 17(11):2767-2779. doi:10.1007/ s00330-007-0651-9

9. Knight JR, Heran M, Munk PL, Raabe R, Liu DM (2008) C-arm cone-beam CT: applications for spinal cement augmentation demonstrated by three cases. J Vasc Interv Radiol 19(7):1118-1122. doi:10.1016/j.jvir.2008.04.001

10. Hiwatashi A, Yoshiura T, Noguchi T, Togao O, Yamashita K, Kamano H, Honda H (2008) Usefulness of cone-beam CT before and after percutaneous vertebroplasty. AJR Am J Roentgenol 191(5):1401-1405. doi:10.2214/AJR.08.1086

11. Johnson C (2005) Measuring pain. Visual analog scale versus numeric pain scale: what is the difference? J Chiropr Med 4(1):43-44. doi:10.1016/S0899-3467(07)60112-8

12. Ruhoy MK, Newberg AH, Yodlowski ML, Mizel MS, Trepman E (1998) Subtalar joint arthrography. Semin Musculoskelet Radiol 2(4):433-438

13. Wiewiorski M, Valderrabano V, Kretzschmar M, Rasch $H$, Markus T, Dziergwa S, Kos S, Bilecen D, Jacob AL (2009) CTguided robotically-assisted infiltration of foot and ankle joints. Minim Invasive Ther Allied Technol 18(5):291-296. doi:10.1080/ 13645700903059193

14. Sofka CM, Adler RS (2002) Ultrasound-guided interventions in the foot and ankle. Semin Musculoskelet Radiol 6(2):163-168. doi: $10.1055 / \mathrm{s}-2002-32362$

15. Berliner L, Buffa A (2011) Super-resolution variable-dose imaging in digital radiography: quality and dose reduction with a fluoroscopic flat-panel detector. Int J Comput Assist Radiol Surg. doi:10. 1007/s11548-011-0545-9

16. Messmer P, Gross T, Suhm N, Regazzoni P, Jacob AL, Huegli RW (2004) Modality-based navigation. Injury 35(Suppl 1):S-A24-A29 